\title{
Functional analysis of two Sp1/Sp3 binding sites in murine Nanog gene promoter
}

\author{
Da Yong $\mathrm{Wu}^{1}$, Zhen Yao $^{1}$ \\ ${ }^{1}$ Laboratory of Molecular Cell Biology, Laboratory of Stem Cell Biology, Institute of Biochemistry and Cell Biology, Shanghai Insti- \\ tutes for Biological Sciences, Chinese Academy of Sciences, 320 Yue Yang Road, Shanghai 200031, China
}

Nanog gene plays a key role in maintaining pluripotency of ES cells and early embryonic cells. A 5' flank sequence of the Nanog gene has been reported to be regulated differentially, and two regulatory elements within the Nanog promoter, namely Oct-4 and Sox-2 binding sites, have been identified to regulate the transcriptional activity of Nanog gene. In this report, we identified the role of two putative Sp1 binding sites located in the Nanog gene 5'-flanking region in regulation of murine Nanog gene transcription. Mutation studies showed that the two sites were essential for the Nanog promoter activity. Gel shift and supershift analysis showed that both sites specifically bind Sp1 and Sp3. Furthermore, overexpression of dominant-negative Sp1 or Sp3 mutants significantly inhibits Nanog promoter activity. These results suggest that the transcription factor $\mathrm{Sp} 1$ and $\mathrm{Sp} 3$ are important for Murine Nanog gene expression.

Cell Research (2006) 16:319-322. doi:10.1038/sj.cr.7310040; published online 16 March 2006

Keywords: Nanog, promoter, Sp1, Sp3

The transcription factor Nanog plays a crucial role in the maintenance of the undifferentiated state of embryonic stem cells $[1,2]$. Like Oct-4, it has been accepted as an important mark for pluripotent cells.

Previous studies suggested that Oct-4 and Nanog work in parallel pathways. The loss of Nanog does not affect oct-4 gene expression, and the loss of oct-4 does not affect Nanog gene expression in the blastocyst [2]. However, there were evidences showing that Oct- 4 is necessary for Nanog gene expression [3], which was confirmed by recent reports that Oct-4 and Sox-2 synergistically activate Nanog gene expression $[4,5]$.

The Oct-4 and Sox-2 binding sites are located within the differentially regulated Nanog promoter region and responsible for its activity [6]. However, overexpression of Oct4 and Sox-2 have little effect on rescuing Nanog promoter activity in differentiated cells [4], indicating that

Correspondence: Da Yong Wu

Tel: 86-21-54921367;

E-mail: dywu@sibs.ac.cn

Received 14 Aug 2005; revised 26 Nov 2005; accepted 20 Dec 2005; published online 16 March 2006 some other transcription factors, besides Oct 4 and Sox2, may be required for the regulation of Nanog gene expression [4]. In this work, we tried to find other cis-elements within the Nanog promoter participating in the Nanog gene transcriptional regulation.

\section{Materials and Methods}

\section{Cell culture and transient transfection}

F9 EC cells were maintained in Dulbecco's modified Eagle's medium (DMEM) (Life Technologies) supplemented with $10 \%$ fetal bovine serum (Hyclone). Cells were seeded at $5 \times 10^{4}$ cells per well in 24 well plates $24 \mathrm{~h}$ prior to transfection. $1 \mu \mathrm{g}$ each of the promoter reporters constructs and $0.02 \mu \mathrm{g}$ pRL-TK (Promega) were co-transfected in duplicate by LipofectAMINE 2000 according to the manufacturer's instructions. Cells were then lysed with $100 \mu \mathrm{l}$ passive lysis buffer (Promega) $24 \mathrm{~h}$ after transfection and the cell lysates were vortexed and briefly centrifuged to sediment cell debris. $20 \mu \mathrm{l}$ aliquot of cell lysate was then assayed for luciferase activities using the Dual Luciferase Reporter (DLR) assay system (Promega) according to the supplier's recommendations. All transfections were repeated three times and shown with representative samples. Plasmid DNA was purified using Qiagen tip-20 column.

Promoter-reporter constructs and site-directed mutagenesis 
Dominant negative $\mathrm{Sp} 1$ and $\mathrm{Sp} 3$ expression constructs were gifts from Prof Thiel G. To create the mutant constructs pNANPmSP1A, pNANPmSP1B and pNANPmSP1AB, the putative Sp1 binding sequence was replaced by introducing Nhe I and Xba I sites with a method based on bridging-based two round PCR [7] with PNANP$220 /+6$ as template. The primers are listed in Table 1 .

Nuclear extract preparation and electrophoretic gel mobility shift assay

Nuclear proteins were extracted from F9 EC cells as previously described [8]. Complementary oligonucleotides (as shown in Table 1) were annealed in annealing buffer ( $10 \mathrm{mM}$ Tris-Cl, $\mathrm{pH} 7.5,1 \mathrm{mM}$ EDTA, $100 \mathrm{mM} \mathrm{NaCl}$ ) and the resulting double-stranded oligodeoxynucleotide (dsODN) probes were end-labeled with $\left[\gamma_{-}{ }^{32} \mathrm{P}\right]$ ATP by T4 polynucleotide kinase (Tarkara). For gel mobility shift assays, 2 $\times 10^{4} \mathrm{cpm}$ probe was incubated with $4 \mu \mathrm{g}$ of nuclear extracts in 20 $\mu l$ volume of binding reaction buffer $(10 \mathrm{mM}$ Tris- $\mathrm{Cl}, \mathrm{pH} 7.5,50$ $\mathrm{mM} \mathrm{NaCl}, 1 \mathrm{mM} \mathrm{MgCl} 2,0.5 \mathrm{mM}$ EDTA, $5 \%$ glycerol, $0.05 \mathrm{mg} / \mathrm{mL}$ Poly (dI/dC)) or sperm DNA on ice for $30 \mathrm{~min} .50$ or 100 fold molar excess of the individual double-stranded oligonecleotides were included in the binding reaction for both specific and non-specific competition analyses. In supershift experiments, Sp1 (PEP-2) or Sp3 (D20) polyclone antibody (Santa Cruz) was incubated with nuclear extracts in a $20 \mu \mathrm{l}$ volume of binding reaction for $30 \mathrm{~min}$ on ice, followed by incubation with the labeled probe on ice for an additional $30 \mathrm{~min}$. Complexes were separated on $4 \%$ non-denaturing polyacrylamide gels. The gels were subsequently dried and autoradiography was performed.

\section{Results and Discussion}

Like many other genes in early embryo as $f g f 4$, sox-2, utfl and $f b x 15$, Nanog gene expression is regulated by Oct-4 and Sox-2 synergistically $[4,5]$. However, overexpression of Oct-4 or Sox-2, or both, could not effectively activate the Nanog gene promoter [5]. Here we further analyzed other putative bindings sites located within the

Table 1 Oligonucleotides used in this study

\begin{tabular}{lll}
\hline Usage & oligonucleotide & sequence \\
\hline Mutant & sense (Site A) & 5'-gctagctctagaggtaggaggcttgaggg-3' \\
constructs & antisense (Site A) & 5'-gtgcctgggagaatagctagctctaga-3' \\
& Sense (Site B) & 5'-gctagctctagaggacctaccctttaa-3' \\
& antisense (Site B) & 5'-gtaggaggcttgaggctagctctaga-3' \\
EMSA & Probe A & 5'-agaatagggggtgggtagggt-3' \\
& & 5'-agaatagggggtgggtagggt-3' \\
& Probe B & 5'-cttgaggggggaggagcaggacc-3' \\
& & 5'-cttgaggggggaggagcaggacc-3' \\
& Consensus & 5'-attcgatcggggcggggcgagc-3' \\
& & 5'-attcgatcggggcggggcgagc-3' \\
& Nonspecific & 5'-cgcttgatgagtcagccggaa-3' \\
& competitor & 5'-cgcttgatgagtcagccggaa-3' \\
\hline
\end{tabular}

same promoter region, which may be responsible for Nanog promoter activity. Two putative Sp1 binding sites centered at -50 and -76 were revealed using TRANSFAC and GENOMATIX softwares. To characterize the functions of those sites, mutation analyses were carried out. The putative Sp1 binding sites centered at -50 and -76 were mutated by replacing the original nucleotides with sequences of restriction enzyme sites that lack any known cis-element. As Figure 1 showed, compared to the wild-type construct, mutation of the site at -76 reduced Nanog promoter activity approximately by $50 \%$, and mutation of the site at -50 decreased the reporter activity by nearly 5 times, suggesting that the two putative Sp1 binding sites might be critical for Nanog expression. Moreover, double mutation of the two sites decreased Nanog promoter activity by nearly 20 times, indicating that the two sites may act synergistically. Although a third putative Sp1 binding site was revealed at -110 by software analyses, mutation of the site did not affect Nanog promoter activity (data not shown).

EMSA was then carried out to search for the proteins binding to the putative Sp1 sites. Oligonucleotides containing the two putative $\mathrm{Sp} 1$ sites were synthesized and labeled with $\left[\gamma^{-32} \mathrm{P}\right]-$ ATP as probes and incubated with F9 cell nuclear extracts. Data showed that three shift bands were formed with either probe (Figure 2A and 2B). The intensity of the three bands were significantly reduced by the addition of $50 \times$ molar excess cold Sp1 oligonucleotide, but not by addition of non specific competitor (Figure 2A and 2B), which confirmed the specificity of the proteins binding to the two putative $\mathrm{Sp} 1$ sites. Supershift experiments showed that addition of $\mathrm{Sp} 1$ antibody inhibited the formation of the upper band, and the two bands below were inhibited by addition of $\mathrm{Sp} 3$ antibody, indicating that the two sites selectively bound Sp1 and Sp3 (Figure 2A and 2C). The sequence at site -50 showed stronger binding affinities to $\mathrm{Sp} 1 / \mathrm{Sp} 3$ than the one at -76 , which is in agreement with the results of above mutation promoter assay, proposing the downstream site might contribute more to the promoter activity than the upstream one.

To further confirm the role of $\mathrm{Sp} 1$ and $\mathrm{Sp} 3$ on the regulation of the Nanog promoter activation, co-transfection experiments with dominant-negative (DN) mutants of Sp1 and $\mathrm{Sp} 3$ were carried out. DN mutants of Sp1 and Sp3 contain intact DNA binding regions, but lack any transcriptional activation function. They could effectively block $\mathrm{Sp} 1$ or Sp3 DNA binding sites [9]. In this experiment, F9 EC cells were co-transfected with the wild type reporter plasmids and expression vectors of dominant negative mutants of Sp1 or Sp3, namely GST-Sp1 or GST-Sp3. As a control, an expression vector encoding a nuclear-targeted glutathione S-transferase (GST) was used. The results showed that expression of either GST- Sp1 or GST- Sp3 resulted in an 
A

CTGGGTGCCT GGGAGAATAG GGGGTGGGTA GGGTAGGAGG CTTGA AGGGGG

$-99$

$\mathrm{Sp} 1(\mathrm{~A}) \quad \mathrm{Sp} 1(\mathrm{~B})$

GAGGAGCAGG ACCTACCCTT TAAATCTATC GCCTTGAGCC GTTGGCCTTC

$+1$

B

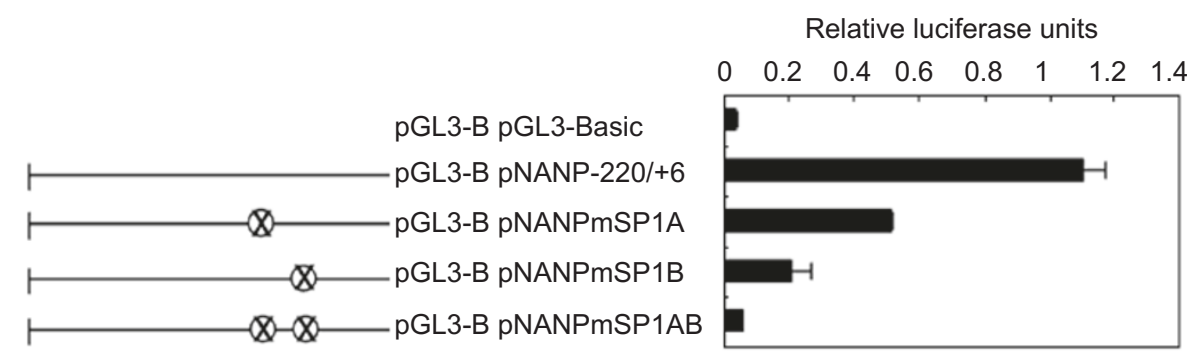

Figure1 (A) Sequence of murine Nanog 5'-flanking region from -99 to +1 . Two putative transcription factor binding sites centered at -50 and -76 as underlined. (B) Role of two sp1/3 binding sites in the expression of the Nanog promoter in F9 EC cells. Duplicate plates of F9 EC cells were transfected with either the wild type or mutagenized Nanog promoter/reporter construct (left) and analyzed for promoter activity (right). Firefly luciferase expression levels were normalized to the luciferase activity of internal Renilla control and expressed as relative luciferase units. The results are the mean \pm S.D. of duplicate from one of three separate experiments.

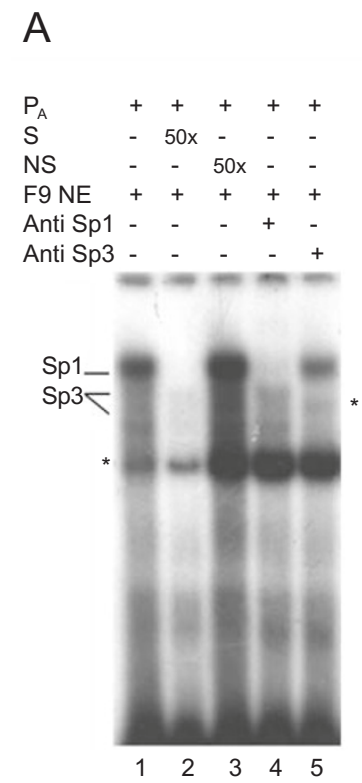

B

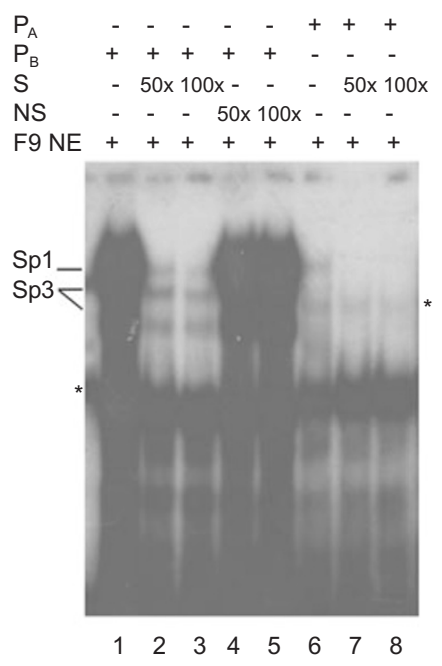

C

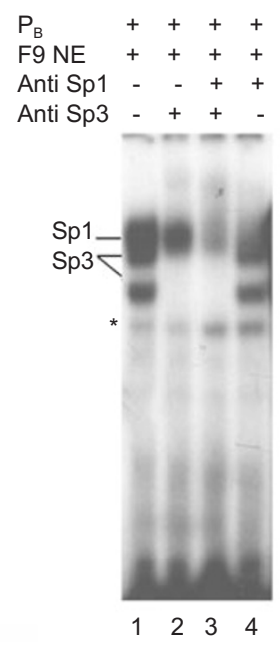

Figure 2 Gel shift analyses of nuclear proteins binding to the $\mathrm{Sp} 1$ probes. $\left.{ }^{32} \mathrm{P}\right]$-labeled Sp1 probes were incubated with nuclear extract proteins from F9 EC cells. Protein-DNA complexes were resolved on 4\% non-denaturing polyacrylamide gel and visualized using autoradiography. (A) Lane 1, Probe A incubated with F9 nuclear extract proteins; lane 2 and 3, specific and nonspecific competition; lane 4 and 5, supershift assays with Sp1 and Sp3 antibody respectively. (B) Lane 1 and 6, Probe B and probe A respectively incubated with F9 nuclear extract proteins; lane 2 and 3 and lane 7 and 8, specific competition; lane 4 and 5, nonspecific competition. (C) Lane 1, Probe B incubated with F9 nuclear extract proteins; lane 2-4, supershift assays with Sp1 antibody, Sp3 antibody and both, respectively. ${ }^{*}$ Represents nonspecific bands; $\mathrm{S}$, specific competitor; NS nonspecific competitor; $\mathrm{P}_{\mathrm{A}}$, probe $\mathrm{A} ; \mathrm{P}_{\mathrm{B}}$, probeB; F9 NE, F9 cell extracts. 
Relative luciferase units

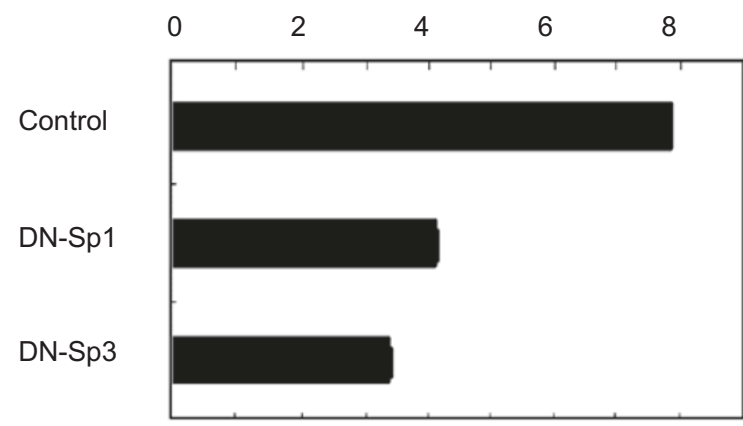

Figure 3 Role of dominant negative of $\mathrm{Sp} 1$ or Sp3 mutants on the expression of the Nanog promoter in F9 EC cells. Duplicate plates of F9 EC cells were co-transfected with dominant negative Sp1 mutant or Sp3 mutant with Nanog promoter/reporter for the analysis of promoter activity. Firefly luciferase expression levels were normalized to the luciferase activity of internal Renilla control and expressed as relative luciferase units. The results are the mean \pm S.D. of duplicate from one of three separate experiments.

obvious decrease in the Nanog promoter activity (Figure $3)$. The inhibitory effects were much more significant in human TERA-2 EC cells, the reason of which may be that the human gene promoter was used to create GST-Sp1 and GST-Sp3. These data indicate that both $\mathrm{Sp} 1$ and Sp3 bind the two putative $\mathrm{Sp} 1$ sites within the Nanog promoter and they may participate in the regulation of the constitutive transcriptional activity of Nanog gene.

$\mathrm{Sp} 1$ and Sp3 are two ubiquitously expressed transcription factors and belong to the Sp1-like/KLF family of the transcription factors. Members of this family are implicated in the regulation of diverse cellular functions $[10,11]$. Sp1 and $\mathrm{Sp} 3$ participate in the activation of oct-4 expression, which is differentially regulated during mouse development [12]. Here we report that they are also involved in murine Nanog gene transcriptional regulation. However, $\mathrm{Sp} 1$ or Sp3 knockout mice were still able to form intact inner cell mass $[13,14]$, suggesting that $S p 1$ and Sp3 have redundant functions that compensate for each other in $\mathrm{Sp} 1$ or Sp3 knockout mice, Perhaps the generation of ES cells with double knockout of Sp1 and Sp3 will unravel the question.

\section{Acknowledgement}

We thank Mrs Xiu Lan Li for her technical supports in this work.

\section{References}

1 Mitsui K, Tokuzawa Y, Itoh H, et al. The homeoprotein Nanog is required for maintenance of pluripotency in mouse epiblast and ES cells. Cell 2003; 113:631-642.

2 Chambers I, Colby D, Robertson M, et al. Functional expression cloning of Nanog, a pluripotency sustaining factor in embryonic stem cells. Cell 2003; 113:643-655.

3 Du Z, Cong H, Yao Z. Identification of putative downstream genes of Oct-4 by suppression-subtractive hybridization.Biochem Biophys Res Commun 2001; 282:701-706.

4 Rodda DJ, Chew JL, Lim LH, et al. Transcriptional regulation of nanog by OCT4 and SOX2. J Biol Chem 2005; 280:24731 $-24737$.

5 Kuroda T, Tada M, Kubota H, et al. Octamer and Sox elements are required for transcriptional cis regulation of Nanog gene expression.Mol Cell Biol 2005; 25:2475-2485.

$6 \mathrm{Wu}$ da Y, Yao Z. Isolation and characterization of the murine Nanog gene promoter. Cell Res 2005; 15:317-324.

$7 \mathrm{Xu} \mathrm{J}, \mathrm{Zhu} J \mathrm{D}, \mathrm{Ni}$ M, Wan F, Gu JR. The ATF/CREB site is the key element for transcription of the human RNA methyltransferase like 1(RNMTL1) gene, a newly discovered $17 \mathrm{p} 13.3$ gene. Cell Res 2002; 12:177-197.

8 Andrews PA, Jones JA. A rapid micropreparation technique for extraction of DNA-binding proteins from limiting numbers of mammalian cells. Nucleic Acids Res 1991; 19:2499.

9 Al-Sarraj A, Day RM, Thiel G. Specificity of transcriptional regulation by the zinc finger transcription factors $\mathrm{Sp} 1, \mathrm{Sp} 3$, and Egr-1.J Cell Biochem 2005; 94:153-167.

10 Bouwman P, Philipsen S. Regulation of the activity of Sp1-related transcription factors. Mol Cell Endocrinol 2002; 195:27-38.

11 Kaczynski J, Cook T, Urrutia R. Sp1- and Kruppel-like transcription factors. Genome Biol 2003; 4:206.

12 Pesce M, Marin Gomez M, Philipsen S, Scholer HR. Binding of $\mathrm{Sp} 1$ and Sp3 transcription factors to the Oct-4 gene promoter. Cell Mol Biol (Noisy-le-grand) 1999; 45:709-716.

13 Bouwman P, Gollner H, Elsasser Hp, et al. Transcription factor $\mathrm{Sp} 3$ is essential for post-natal survival and late tooth development. EMBO J 2000; 19:655-661.

14 Marin M, Karis A, Visser P, Grosveld F, Philipsen S. Transcription factor $\mathrm{Sp} 1$ is essential for early embryonic development but dispensable for cell growth and differentiation. Cell 1997; 89: 619-628.

Edited by Duanqing Pei 